\title{
A Difficult Case of Necrotizing Fasciitis Caused By Acinetobacter baumannii
}

The International Journal of Lower Extremity Wounds

9(4) 152-154

(C) The Author(s) 2010

Reprints and permission: http://www. sagepub.com/journalsPermissions.nav DOI: 10.1 | 777/I5347346/0389598 http://ijlew.sagepub.com

@SAGE

\author{
Bartolo Corradino, MD', Francesca Toia, MD', Sara di Lorenzo, MD', \\ Adriana Cordova, MD', and Francesco Moschella, MD'
}

\begin{abstract}
This study reports the case of a 55-year-old woman with diabetes with a necrotizing fasciitis of the right lower limb and the perineum, first admitted at the emergency department for septic shock with cardiac arrest, and later transferred to the department of surgery. Microbiological and histopathological examination confirmed the diagnosis of necrotizing fasciitis caused by Acinetobacter baumanii.A broad-spectrum antibiotic therapy was administered and later readjusted according to the results of microbiological culture. Intensive hemodynamic support was required. Wounds were dressed daily with a 3 percent boric acid solution and a silver sulfadiazine-impregnated dressing. An extensive surgical debridement was promptly performed and repeated until complete control of the infection. Wounds were finally covered with split-thickness skin grafts. The infection was overcome 35 days after admission. The graft take was 100\%. Postoperative rehabilitation was required because of the functional limitation of lower limb movements. Follow-up at 6 months showed no functional deficit and an acceptable aesthetic result. Necrotizing fasciitis is a life-threatening disorder, especially in patients with diabetes, whose clinical diagnosis may sometimes be challenging. Early recognition and treatment represent the most important factors influencing survival.
\end{abstract}

\section{Keywords}

necrotizing fasciitis, Acinetobacter baumanni, soft tissue infection

\section{Case Report}

Necrotizing fasciitis is an uncommon soft-tissue infection, associated with a high morbidity and mortality. ${ }^{1,2}$ Its clinical diagnosis may be challenging. Early recognition and treatment represent the most important factors influencing survival. ${ }^{2}$ Acinetobacter baumannii is rarely associated with necrotizing fasciitis, especially in immunocompetent hosts. ${ }^{3}$

We report the case of a 55-year-old woman first admitted at the emergency department in this center for septic shock with cardiac arrest. The patient had no history of previous hospitalization. She reported a history of local erythema and swelling of the right thigh 1 month previously, rapidly evolving in the 3 days before admission into extensive necrosis of skin and subcutaneous tissue of the whole lower limb and the perineum. Purulent discharge was present, and a broadspectrum antibiotic therapy was administered. Microbiological cultures revealed an infection by Acinetobacter baumannii, but some days later the patient acquired a super-infection by Pseudomonas aeruginosa. Nine days later, she was transferred to our department.

Her previous medical history included hypertension, cigarette smoking, and chronic obstructive pulmonary disease; diabetes mellitus was diagnosed during hospitalization. Alcohol abuse was excluded.
Laboratory data on admission showed a white blood cell count of $22.26 \times 10^{3} \mathrm{~mm}^{-3}$, hemoglobin $7.8 \mathrm{~g} / \mathrm{dL}$, glucose blood level $155 \mathrm{mg} / \mathrm{dL}$, hypoalbuminemia, and hydroelectrolytic disorders. A fever $\left(38.8^{\circ} \mathrm{C}\right)$, a pleuric-pericardial effusion, and a hepatosplenomegaly were also present. Serologic studies excluded an infection by HIV.

Diffuse erythema and swelling of the lower limb and perineum was noted, together with a severe hypotonia and hypotrophy of the limb. The skin was partially necrotic; fistulous tracts and purulent discharge were present. (Figure 1) No crepitus was noted. X-ray was not significant for soft tissue air.

A working diagnosis of necrotizing fasciitis was made. Intensive hemodynamic support was required and specific antibiotic therapy was administered. The patient was given intravenous gentamycin $80 \mathrm{mg}$ every 8 hours and intravenous ciprofloxacin $400 \mathrm{mg}$ every 12 hours. An extensive surgical debridement was promptly performed. Multiple

'Dipartimento di Discipline Chirurgiche e Oncologiche. Sezione di Chirurgia Plastica. Università degli Studi di Palermo, Italy

\section{Corresponding Author:}

Francesca Toia, Dipartimento di Discipline Chirurgiche e Oncologiche, Sezione di Chirurgia Plastica, Università degli Studi di Palermo, Via del Vespro, I29, 90I27 Palermo, Italy

Email: francytoia@inwind.it 


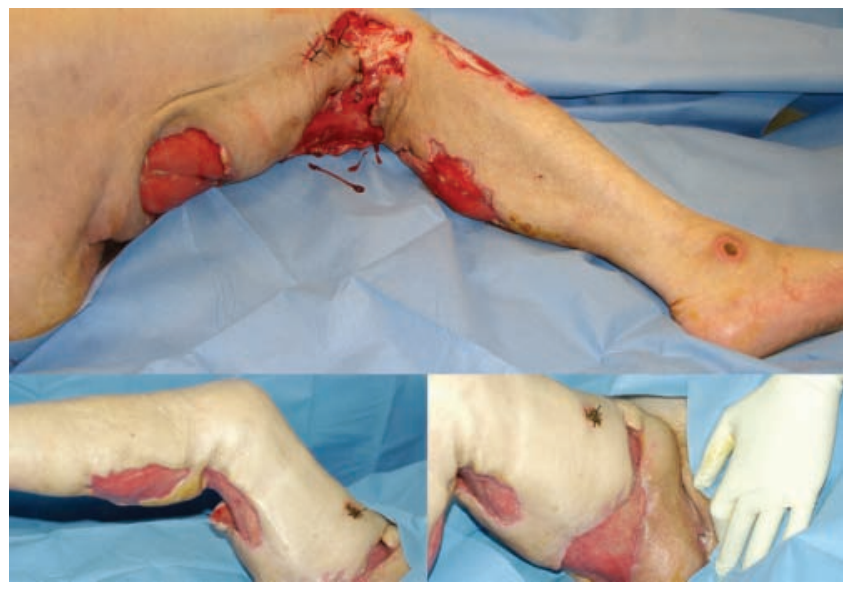

Figure I. Aspect of the patient after initial debridement.

cultures were taken and set for aerobic and anaerobic cultures, and biopsy specimens were sent for histopathological examination.

In the following days, blood transfusions were necessary and the patient was put in enteral nutrition. Because of the worsening general conditions, it was not possible to perform further aggressive surgical debridement; the patient was also not suitable for hyperbaric oxygen therapy. Wounds were dressed daily with a $3 \%$ boric acid solution and silver sulfadiazine and minor debridement was performed at the bedside. Physiotherapy was initiated promptly.

Microbiological and histopathological examination confirmed the diagnosis of necrotizing fasciitis. $P$ aeruginosa and $A$ baumannii were isolated from both wounds and blood, and antibiotic therapy was rearranged as dictated by culture antibiogram results.

Antibiotic therapy was continued for 5 weeks and, because of progressive antimicrobial resistance, required substitution of gentamycin by amikacin $1 \mathrm{~g} / \mathrm{d}$ on day 8 after admission, and of ciprofloxacin for colymicin $\left(1 \times 10^{6} \mathrm{U}\right.$ intramuscular every 12 hours) on day 17.

Further surgical debridement was performed according to general conditions of the patient, on days 9, 19, and 31 . Bedside debridement was performed daily during the whole period of hospitalization. On day 22, patient suffered acute renal failure, which complicated the treatment.

Thirty-five days after admission, the infection was overcome. Fistulous tracts were closed primarily, and wounds were finally covered with split-thickness skin grafts in 2 different operations. Physiotherapy was interrupted for 7 days following skin grafting. The graft take was $100 \%$ and on day 45 the patient was discharged (Figure 2).

Postoperative rehabilitation was required because of the functional limitation of lower limb movements. Follow-up at 6 months showed no functional deficit and an acceptable aesthetic result.

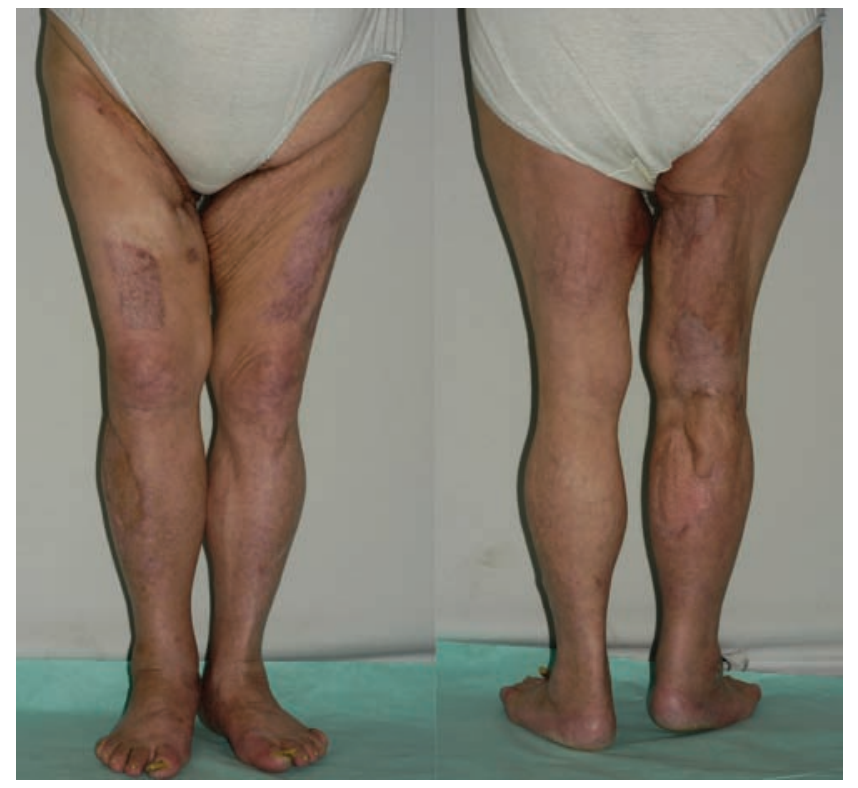

Figure 2. Six-months postoperative aspect.

\section{Discussion}

Necrotizing fasciitis is a life-threatening disorder, whose early recognition is often difficult and has an effect on survival. ${ }^{1}$ It is classically classified into 2 subtypes. Type I infections are polymicrobial and account for the most common form (55\% to $75 \%)$. Type II infection results from a group A Streptococcus alone or in association with Staphylococcus aureus. ${ }^{4}$ Some reports describe a type III infections, those caused by Vibrio vulnificus. ${ }^{5}$ Predisposing factors include immunosuppression, diabetes, obesity, malnutrition, and peripheral vascular disease. ${ }^{1}$

Acinetobacter baumannii is rarely encountered as pathogen causing soft-tissue infection, especially in immunocompetent hosts, and can rarely be responsible for community-acquired necrotizing fasciitis. ${ }^{3}$

In the case we reported, uncontrolled diabetes and cigarette smoking were the only risk factors present in this otherwise healthy patient, and no history of previous hospitalization was reported. A super-infection by $P$ aeruginosa complicated the course.

Therapeutic management of necrotizing fasciitis requires a comprehensive approach, the cornerstone of therapy being a rapid and extensive surgical debridement. Adequate wound dressing management and antibiotic therapy are also essential. ${ }^{2}$ As in severe burns, the extent of skin and soft tissue defects are responsible for systemic perturbations, which require an intensive supportive care.

When general condition of the patient is poor, it is often not possible to perform serial extensive debridement in the operating room. In these cases, surgical operations can be alternated with bedside debridement. When clinical conditions permit, 
hyperbaric oxygen therapy can contribute to healing and should be considered as an option. Reconstructive surgery is almost always necessary, and marked sequelae persist. Postoperative rehabilitation is often necessary for functional recovery.

\section{Declaration of Conflicting Interests}

The author(s) declared no potential conflicts of interests with respect to the authorship and/or publication of this article.

\section{Funding}

The author(s) received no financial support for the research and/ or authorship of this article.

\section{References}

1. Cheung JP, Fung B, Tang WM, Ip WY. A review of necrotising fasciitis in the extremities. Hong Kong Med J. 2009;15: 44-52.
2. Gürlek A, Firat C, Oztürk AE, Alaybeyoglu N, Fariz A, Aslan S. Management of necrotizing fasciitis in diabetic patients. $J$ Diabetes Complications. 2007;21:265-271.

3. Chiang WC, Su CP, Hsu CY, et al. Community-acquired bacteremic cellulitis caused by Acinetobacter baumannii. J Formos Med Assoc. 2003;102:650-652.

4. Swartz MN, Pasternack MS. Cellulitis and subcutaneous tissue infections. In: Mandell GL, Bennett JE, Dolin R, eds. Principles and Practice of Infectious Diseases. 6th ed. Philadelphia, PA: Churchill Livingstone; 2005:1172-1194.

5. Sarani B, Strong M, Pascual J, Schwab CW. Necrotizing fasciitis: current concepts and review of the literature. J Am Coll Surg. 2009;208:279-288. 